\title{
CARACTERIZAÇÃO SOCIOECONÔMICA E LEVANTAMENTO DE ESPÉCIES VEGETAIS EM QUINTAIS AGROFLORESTAIS DA ZONA RURAL DO MUNICÍPIO DE PARAUAPEBAS, PARÁ
}

\author{
Gleiciane Cardoso Costa; Nayara Dayane Soares Moura²; Ana Karolina Dias Farias; \\ Erondina Araújo Alho ${ }^{4}$; Gládis de Oliveira Jucoski ${ }^{5}$. \\ ${ }^{1}$ Graduanda em Eng. Florestal, Universidade Federal Rural da Amazônia (UFRA), Parauapebas, Pará, Brasil, \\ gleiciane.gc9@gmail.com \\ ${ }^{2}$ Graduanda em Eng. Florestal, UFRA, Parauapebas, Pará, Brasil, nayara.moura26@gmail.com \\ ${ }^{3}$ Graduanda em Eng. Florestal, UFRA, Parauapebas, Pará, Brasil, karolinadias12@gmail.com \\ ${ }^{4}$ Graduanda em Eng. Florestal, UFRA, Parauapebas, Pará, Brasil, erondinaalho@hotmail.com \\ ${ }^{5}$ Professora da UFRA, Parauapebas, Pará, Brasil, jucoski@yahoo.com.br
}

RESUMO: Objetivou-se realizar a caracterização socioeconômica e o levantamento das espécies vegetais que compõem a biodiversidade dos quintais agroflorestais rurais do município de Parauapebas-Pa. Realizou-se o estudo em 6 quintais agroflorestais localizados na zona rural do município nos bairros Palmares I e Vs 10. Foi utilizado o método turnêguiada, e a pesquisa de campo ocorreu em duas etapas. Sendo a primeira com aplicação de questionários socioeconômicos, e a segunda com a aplicação de uma ficha de campo para coleta de dados relativos as espécies cultivadas. Os parâmetros ecológicos abordados foram a abundância, riqueza, e o índice de diversidade de Shannon-Wiener $\left(\mathrm{H}^{\prime}\right)$. Os dados mostraram que a principal motivação para manter o quintal é pela segurança alimentar (70\%), a colheita ocorre de forma manual em 83\% dos casos, e cerca de 70\% da adubação dos cultivos é orgânica. Em relação as espécies vegetais, foram identificadas 42 famílias botânicas, 79 gêneros, com a riqueza de 89 espécies e 908 indivíduos. A família Malvaceae apresentou maior abundância com 142 indivíduos, e o índice Shannon-Wiener encontrado para os quintais agroflorestais variou de 2,21 a 3,45. Desse modo, os quintais presentes no município promovem a segurança alimentar, e também são áreas de conservação da biodiversidade local.

PALAVRAS-CHAVE: Agroecossistemas, Agricultura familiar, Segurança alimentar.

\section{SOCIOECONOMIC CHARACTERIZATION AND SURVEY OF PLANT SPECIES IN AGROFORESTRY HOMEGARDENS IN THE RURAL AREA OF THE MUNICIPALITY OF PARAUAPEBAS, PARÁ}

ABSTRACT: The objective was to carry out the socioeconomic characterization and the survey of the vegetal species that compose the biodiversity of the rural agroforestry homegardens in the municipality of Parauapebas-Pa. The study was carried out in 6 agroforestry homegardens located in the rural area of the municipality in the neighborhoods Palmares I and Vs 10. The guided excursions method was used, and field 
research occurred in two stages. Being the first one with the application of socioeconomic questionnaires, and the second with the application of a field record for data collection on the cultivated species. The ecological parameters addressed were the abundance, richness, and diversity index of Shannon-Wiener ( $\left(H^{\prime}\right)$. The data showed that the main motivation to maintain the homegardens is food security (70\%), the harvest occurs manually in $83 \%$ of the cases, and about $70 \%$ of the fertilization of the crops is organic. Regarding plant species, 42 botanical families were identified, 79 genera, with a richness of 89 species and 908 individuals. The Malvaceae family showed greater abundance with 142 individuals, and the Shannon-Wiener index found for agroforestry gardens ranged from 2.21 to 3.45. In this way, the homegardens present in the municipality promote food security, and are also areas of conservation of local biodiversity.

KEYWORDS: Agroecosystems, Family agriculture, Food security.

\section{CARACTERIZACIÓN SOCIOECONÓMICA Y LEVANTAMIENTO DE ESPECIES VEGETALES EN QUINTAIS AGROFLORESTAIS DE LA ZONA RURAL DEL MUNICIPIO DE PARAUAPEBAS, PARÁ}

RESUMEN: Se objetivó realizar la caracterización socioeconómica y el levantamiento de las especies vegetales que componen la biodiversidad de los quintos agroforestales rurales del municipio de Parauapebas-Pa. Se realizó el estudio en 6 quintales agroforestales ubicados en la zona rural del municipio en los barrios Palmares I y Vs 10. Se utilizó el método giraguiado, y la investigación de campo ocurrió en dos etapas. Siendo la primera con aplicación de cuestionarios socioeconómicos, y la segunda con la aplicación de una ficha de campo para la recolección de datos relativos a las especies cultivadas. Los parámetros ecológicos abordados fueron la abundancia, riqueza, y el índice de diversidad de Shannon-Wiener ( $\mathrm{H}^{\prime}$ ). Los datos mostraron que la principal motivación para mantener el patio trasero es por la seguridad alimentaria (70\%), la cosecha ocurre de forma manual en el $83 \%$ de los casos, y cerca del $70 \%$ de la fertilización de los cultivos es orgánica. En relación a las especies vegetales, se identificaron 42 familias botánicas, 79 géneros, con la riqueza de 89 especies y 908 individuos. La familia Malvaceae presentó mayor abundancia con 142 individuos, y el índice Shannon-Wiener encontrado para los quintos agroforestales varió de 2,21 a 3,45. De este modo, los quintiles presentes en el municipio promueven la seguridad alimentaria, y también son áreas de conservación de la biodiversidad local.

PALABRAS CLAVE: Agroecosistemas, Agricultura familiar, Seguridad alimentaria. 
Uma alternativa para o manejo sustentável do solo é a implantação de sistemas agroflorestais nas propriedades agrícolas. Segundo Castro et al. (2009) os sistemas agroflorestais estão representados por vários sistemas de uso da terra, como: silviagrícola, onde estão associados árvores ou arbustos com cultivos agrícolas anuais e/ou perenes; agropastoril, cultivos agrícolas perenes associados com atividade pecuária; silvipastoril, árvores associadas com atividade pecuária; e, agrossilvipastoril, árvores associadas com cultivos agrícolas e atividade de pecuária.

Dentre os sistemas agroflorestais, os quintais ou hortos caseiros, por suas características ecológicas e socioeconômicas, são especialmente adequados quando se busca estabilidade produtiva no âmbito da unidade familiar. Nos quintais agroflorestais, espécies agrícolas e florestais são conjugadas buscando-se otimizar o uso dos recursos naturais por meio de interações positivas entre os componentes vegetais e/ou animais com baixo uso de insumos. Nessas áreas, localizadas próximas às residências, são obtidos produtos alimentícios, medicinais e para outros fins, fortemente voltados para o autoconsumo familiar (GOMES, 2010).

Conforme Amaral (2008), os quintais são sistemas agroflorestais que desempenham função ecológica, conservam alta diversidade de plantas na sua composição, asseguram variabilidade genética, constituindo importantes bancos de gemoplasma, representando sistemas sustentáveis com maior resistência a doenças e adaptabilidade.

Esses agroecossistemas demandam baixos insumos e representam uma fonte adicional de renda, caracterizando-se como uma atividade potencial para a obtenção de alimentos e para suprir as necessidades de lenha e madeira para consumo da família. Esse espaço produtivo permite que a unidade de produção familiar garanta a segurança alimentar da família, além de ser uma alternativa economicamente viável para OS agricultores familiares (FIGUEIREDO JÚNIOR et al., 2013).

Para Gazel Filho (2008) estudos sobre a composição botânica e a estrutura dos quintais e das propriedades de suas espécies podem fornecer informações 
básicas para tomadas de decisões na aplicação de técnicas de intensificar e melhorar o manejo da terra ou conservação destes espaços.

Dessa maneira, esse trabalho tem por objetivo realizar a caracterização socioeconômica e o levantamento das espécies vegetais que compõem a biodiversidade dos quintais agroflorestais rurais do município de Parauapebas, Pará.

O estudo foi realizado no município de Parauapebas $06^{\circ} 3^{\prime \prime}$ de latitude sul, e $49^{\circ} 5^{\prime \prime}$ de longitude oeste de Greenwich, localizado no sudeste do Pará.

O município está localizado na chamada "Zona Tropical", apresentando dois subtipos de clima, o de planícies e o de montanhas, ambos de acordo com a classificação do Köppen, clima "Am" tropical, quente e úmido, com precipitação elevada. A estação seca ocorre entre os meses de maio e novembro. No período de chuvas, a precipitação pode alcançar 2800 mm e a umidade relativa do ar chega a ultrapassar 90\%. A temperatura média ao longo do ano é de $29^{\circ} \mathrm{C}$ (SIQUEIRA et al., 2012).

O levantamento foi realizado em seis quintais agroflorestais localizados na zona rural do município nos bairros Palmares I e Vs, selecionando-se para a pesquisa os quintais com maior biodiversidade aparente, no qual verificou-se interesse pelos proprietários em participar da pesquisa

Para a realização do estudo foi considerado quintal agroflorestal todo um espaço em torno da residência onde são cultivadas espécies arbóreas, frutíferas, hortaliças, medicinais, ornamentais e/ou com a presença de pequenos animais, conforme Pereira e Figueiredo Neto (2015).

Foi utilizado o método turnê-guiada, no qual o proprietário foi convidado a fazer uma caminhada pelo quintal durante a entrevista, fornecendo informações específicas sobre as práticas de manejo e as espécies vegetais presentes (ALBUQUERQUE; LUCENA, 2004).

A pesquisa de campo foi realizada em duas etapas. Na primeira etapa realizouse a aplicação dos questionários socioeconômicos com os responsáveis pela manutenção das práticas em suas respectivas propriedades. questionamentos envolveram perguntas 
de caráter socioeconômico e perguntas específicas acerca das práticas de manejo dos quintais.

Na segunda etapa foi realizada a aplicação de uma ficha de campo para coleta de dados relativo as espécies cultivadas. Na descrição das espécies verificaram-se as características de uso alimentar, medicinal, florestal (madeireiro e não madeireiro), condimentos, ornamental e sombreamento. Posteriormente a coleta de dados das espécies vegetais, foram realizadas a identificação e classificação taxonômica de acordo com literatura atualizada.

Os parâmetros ecológicos abordados neste estudo foram: a abundância, riqueza, e o índice de diversidade de Shannon-Wiener $\left(H^{\prime}\right)$. A abundância é a frequência de indivíduos de cada espécie em relação ao total de indivíduos. A riqueza refere-se à abundância numérica de espécies de uma determinada área geográfica, região ou comunidade (RICKLEFS, 2010).

O índice de diversidade de ShannonWiener $\left(\mathrm{H}^{\prime}\right)$ expressa a riqueza de uma amostra ou comunidade e assume que todas as espécies estão representadas, seu valor encontra-se entre 1,5 e 3,5; embora casos excepcionais possam exceder a 4,5 (REZENDE, 2002).

Verificou-se que o tempo médio de moradia dos entrevistados na propriedade é de 12,5 anos. Entre os entrevistados, 50\% exercem atividades profissionais remuneradas, obtendo renda mensal familiar de 1 a 2 salários mínimos.

Para os proprietários a principal motivação para manter os quitais agroflorestais é pela segurança alimentar (70\%). Isso porque, os quintais são reconhecidos como de inestimável valor em áreas rurais, desempenhando importante papel na promoção da segurança alimentar e geração de renda para as famílias.

No que se refere aos cuidados com este subsistema, verificou-se que há participação do homem e da mulher nas atividades de plantio, adubação, irrigação, colheita, trato dos animais e vendas em que ambos estão envolvidos em 50\% das atividades.

Sousa et al. (2014) verificaram em estudos realizados em quintais agroflorestais em Caxias no Maranhão 
que as mulheres são as principais responsáveis pela manutenção e manejo dos quintais. Pereira e Figueiredo Neto (2015) obtiveram resultados semelhantes, no qual demonstram que a mulher desempenha um papel importante na condução do quintal agroflorestal, segundo estes autores a mulher tem participação intensa no manejo do quintal, representando grande força de trabalho na unidade familiar, pois além das atividades produtivas, ela ainda realiza tarefas domiciliares, que englobam diversos serviços domésticos, inclusive a formação e manutenção dos quintais.

Observou-se que o manejo nos quintais visitados, envolve processos simples, de baixo custo, com práticas tradicionais de cultivo, pois a principal força de trabalho na colheita de espécies alimentícias é realizada de forma manual (83\%), e a adubação dos cultivos é realizada principalmente pela adubação orgânica (70\%). Porém, a maioria dos entrevistados (70\%) afirmou utilizar pesticidas para o controle de pragas dos cultivos, devido ao desconhecimento de alternativas ecológicas para o combate das pragas.
Observou-se que todos os quintais agroflorestais visitados apresentam animais como, galinhas (Gallus gallus domesticus), e/ou bovinos (Bos taurus), representando $65 \%$ dos quintais com aves e 35\% com bovinos. No qual, realiza-se o consumo dos ovos e venda das galinhas nas feiras locais e a produção de leite bovino para consumo familiar.

Em relação ao levantamento das espécies cultivadas, observou-se que nos quintais visitados as plantas presentes organizaram-se basicamente em dois componentes, o arbóreo e não arbóreo (arbustivo e estrato herbáceo), sendo classificadas de acordo com a sua principal forma de utilização, como alimentar, medicinal, florestal (madeireiro e não madeireiro), condimentos, ornamental e sombreamento.

No levantamento foram identificadas 42 famílias botânicas, 79 gêneros, com a riqueza de 89 espécies e 908 indivíduos. Quanto ao tipo de uso das espécies temse a seguinte distribuição: alimentar (51), medicinal (13), ornamental (08), madeireira (03), não-madereira (1) sombreamento (09) e condimentos (04) (Tabela 1 e Figura 1). 
Tabela 1. Espécies vegetais presentes nos quintais agroflorestais rurais e seus respectivos usos, Parauapebas, PA, 2017.

\begin{tabular}{|c|c|c|c|}
\hline Família & Nome científico & Nome popular & USO \\
\hline Alliaceae & Allium fistulosum $\mathrm{L}$ & Cebolinha & $A L$ \\
\hline \multirow{4}{*}{ Anacardiaceae } & Spondias monbin L.. & Cajazeiro & $A L$ \\
\hline & Mangifera indica $\mathrm{L}$ & Mangueira & $A L$ \\
\hline & Anacardium occidentale L & Cajueiro & $A L$ \\
\hline & Spondias purpurea L. & Siriguela & $A L$ \\
\hline \multirow{3}{*}{ Annonaceae } & Rollinia mucosa (Jacq.) Baill & Birimbazeiro & $\mathrm{AL}$ \\
\hline & Annona muricata $L$ & Graviola & $A L$ \\
\hline & Annona squamosa $\mathrm{L}$... & Ata & $A L$ \\
\hline Apiaceae & Coriandrum sativum L.. & Coentro & $\mathrm{AL}$ \\
\hline \multirow{5}{*}{ Arecaceae } & Cocos nucifera L.... & Coqueiro & $A L$ \\
\hline & Syagrus oleraceae L. & Guariroba & so \\
\hline & Orbignya phalerata Mart... & Babaccu & $\mathrm{SO}$ \\
\hline & Mauritia flexuosa L.f.... & Buriti & SO \\
\hline & Euterpe oleraceae Mart. & Acaizeiro & $\mathrm{AL}$ \\
\hline \multirow{3}{*}{ Asteraceae } & Chrysanthemum spp. & Crisântemo & OR \\
\hline & Chicorium intybus L... & Chicória & MED \\
\hline & Helianthus annuus L. & Girassol & $\mathrm{OR}$ \\
\hline \multirow{2}{*}{ Bignoniaceae } & Crescentia cujete L. & Cujubeiro & $A L$ \\
\hline & Handroanthus spp. & Ipê & MAD \\
\hline Bixaceae & Bixa orellana $L$ & Urucum & CON \\
\hline Brassicaceae & Brassica oleracea $\mathrm{L}$. & Couve & $\mathrm{AL}$ \\
\hline Bromeliaceae & Ananas comosus (L.) Merr. & Abacaxi & $A L$ \\
\hline Cactaceae & Opuntia cochenillifera (L.) Salm-Dyck. & Cacto & OR \\
\hline Caricaceae & Carica papaya L. & Mamoeiro & $A L$ \\
\hline Cecropiaceae & Cecropia pachystachya Trécul. & Embaúba & $\mathrm{SO}$ \\
\hline Chenopodiaceae & Chenopodium ambrisioides $\mathrm{L}$... & Matruz & MED \\
\hline \multirow{3}{*}{ Cucurbitaceae } & Cucumis sativus $L$ & Pepineiro & $\mathrm{AL}$ \\
\hline & Cucumis anguria $\mathrm{L}$... & Maxixe & $A L$ \\
\hline & Cucurbita spp. & Abóbora & $A L$ \\
\hline \multirow[t]{2}{*}{ Euphorbiaceae } & Manihot esculenta $\mathrm{L}$. & Mandioca & $\mathrm{AL}$ \\
\hline & Ricinus communis L.... & Mamoneira & MED \\
\hline \multirow{6}{*}{ Fabaceae } & Bauhinia forticata L... & Pata de vaca & MED \\
\hline & Inga edulis Mart. & Ingazeiro & $A L$ \\
\hline & Peltophorum dubium (Spreng.) Taub. & Faveiro & $\mathrm{AL}$ \\
\hline & Cenostigmatocantinum Ducke. & Pau Preto & $\mathrm{sO}$ \\
\hline & Parkia pendula (Willd.) Benth. ex Walpers. & Visgeiro & MAD \\
\hline & Tamarindus indica $\mathrm{L}$... & Tamarindo & $A L$ \\
\hline Homoriopsidaceae & Nephrolepsis exalta (L) Schott. & Samambaia & OR \\
\hline Iridaceae & Neomarica gracilis (Herb.) Sprague. & Iris da praia & OR \\
\hline \multirow[t]{2}{*}{ Lamiaceae } & Mentha villosa Huds.... & Menta & MED \\
\hline & Plectranthus barbatus Andrews. & Boldo & MED \\
\hline Lauraceae & Persea americana Miller. & Abacate & $A L$ \\
\hline Lecythidaceae & Bertholletia excelsa Humb. \& Bonpl... & Castanheira & MAD \\
\hline Liliaceae & Aloe vera (L.) Burm.f. & Babosa & MED \\
\hline Lythraceae & Punica granatum $L$.... & Romãnzeira & MED \\
\hline \multirow{2}{*}{ Malpighiaceae } & Malpighia emarjinata $L .$. & Aceroleira & $A L$ \\
\hline & Byrsonima basiloba A. Juss. & Murici & $A L$ \\
\hline \multirow{4}{*}{ Malvaceae } & Pachira aquatica Aulb. & Cacau-Bravo & $\mathrm{AL}$ \\
\hline & Hibiscus sabdafira $L$.... & Vinagreira & $\mathrm{AL}$ \\
\hline & Guazuma ulmifolia Lam. & Mutamba & $A L$ \\
\hline & Gossypium L. & Algodoeiro & $\mathrm{AO}-\mathrm{MA}$ \\
\hline
\end{tabular}




\begin{tabular}{|c|c|c|c|}
\hline & Abelmoschus esculentas L.. & Quiabeiro & $\mathrm{AL}$ \\
\hline & Theobroma grandiflorum (Willd. ex Spreng.) K. Schum. & Cupuaçu & $A L$ \\
\hline & Theobroma cacao $L$ & Cacaueiro & $A L$ \\
\hline & Hibiscus rosa senensis L... & Ibisco & $\mathrm{OR}$ \\
\hline \multirow{4}{*}{ Moraceae } & Manclura tinctoria (L.) Don ex Steud. & Moreira & $\mathrm{SO}$ \\
\hline & Ficus gomelleira Kunth \& C.D.Bouché. & Gameleira & $\mathrm{SO}$ \\
\hline & Artocarpus heterophyllus Forst. & Jagueira & $A L$ \\
\hline & Morus nigra L. & Amora & $\mathrm{AL}$ \\
\hline Musaceae & Musa spp. & Bananeira & $A L$ \\
\hline \multirow[b]{5}{*}{ Myrtaceae } & Psidium guajava $L$... & Goiabeira & $\mathrm{AL}$ \\
\hline & Plinia cauliflora (DC.) O. Berg. & Jabuticabeira & $A L$ \\
\hline & Syzigium malaccense L. & Jambeiro & $A L$ \\
\hline & Pisidium catteleianum Sabine. & Piroqueiro & $A L$ \\
\hline & Syzigium cumini (L.) Skeels. & Azeitona-Preta & $\mathrm{AL}$ \\
\hline Ochnaceae & Ouratea catanefolia (DC.) Engl. & Farinha Seca & SO \\
\hline Oxalidaceae & Averrhoa carambola $\mathrm{L}$... & Caramboleira & $\mathrm{AL}$ \\
\hline Papaveraceas & Papaver somniferum L. & Papoula & $\mathrm{OR}$ \\
\hline Passifloraceae & Passifloraspp. & Maracujá & $A L$ \\
\hline \multirow{3}{*}{ Poaceae } & Saccharum spp. & Cana de açúcar & $A L$ \\
\hline & Cymbopogon citratus Stapf. & Capim-santo & MED \\
\hline & Zea mays $L$ & Milho & $\mathrm{AL}$ \\
\hline \multirow{2}{*}{ Rosaceae } & Rosaxgrandiflora Hort. & Roseira & OR \\
\hline & Prunus salicina L... & Ameixeira & $A L$ \\
\hline \multirow{2}{*}{ Rubiaceae } & Genipa americana L.. & Genipapo & $A L$ \\
\hline & Morinda citrifolia L..... & Planta None & MED \\
\hline \multirow{3}{*}{ Rutaceae } & Citrus sinensis L. Osbeck & Laranjeira & $A L$ \\
\hline & Citrus limonia Osbeck & Limoeiro & $A L$ \\
\hline & Citrus reticulata Blanco. & Tangerina & $A L$ \\
\hline Sapindaceae & Talisia esculenta Radlik... & Pitombeira & $A L$ \\
\hline Sapotaceae & Pouteria macrophylla (Lam.) Eyma. & Tuturubá & $A L$ \\
\hline \multirow{4}{*}{ Solanaceae } & Capsicum frutescens L. & Pimenta malagueta & CON \\
\hline & Capsicum chinense L... & Pimenta de cheiro & $\mathrm{CON}$ \\
\hline & Capsicum baccatum L... & Pimenta dedo de moça & $\mathrm{CON}$ \\
\hline & Solanum lycopersicum Mill. & Tomate & $\mathrm{AL}$ \\
\hline Urticaceae & Parietaria officinalis L.. & Alfava & $\mathrm{ME}$ \\
\hline \multirow[t]{2}{*}{ Verbenaceae } & Lippia alba (Mill)N.E Brown & Erva-cidreira & $\mathrm{ME}$ \\
\hline & Stachytarpheta cayennensis (Rich.) Vahl. & Gervão & ME \\
\hline
\end{tabular}

$\mathrm{AL}=$ Alimentar, $\mathrm{ME}=$ Medicinal, $\mathrm{CON}=$ Condimento $\mathrm{ORN}=$ Ornamental $\mathrm{SO}=$ Sombreamento, $\mathrm{MAD}=$ Madereiro e NÃO-MAD= Não- madeireiro.

Constatou-se que as espécies de uso alimentar representam $57,3 \%$ da população total amostrada, sendo que as espécies frutíferas são as mais abundantes. Para Saviero et al. (2011) estas espécies são responsáveis por garantir a qualidade da alimentação da família por contribuírem para uma alimentação mais saudável.

Garcia et al. (2015) em estudo realizado sobre quintais agroflorestais e segurança alimentar no município de Santarém, constatou que 53\% dos agricultores realizavam autoconsumo das 
espécies produzidas, especialmente as frutas, pois de acordo com os autores as espécies frutíferas são fontes de proteínas e contribuem para uma diversificação e complementação na dieta familiar.

Figura 1. Distribuição das espécies de acordo com a classificação de uso principal dos quintais agroflorestais do município de Parauapebas, PA, 2017.

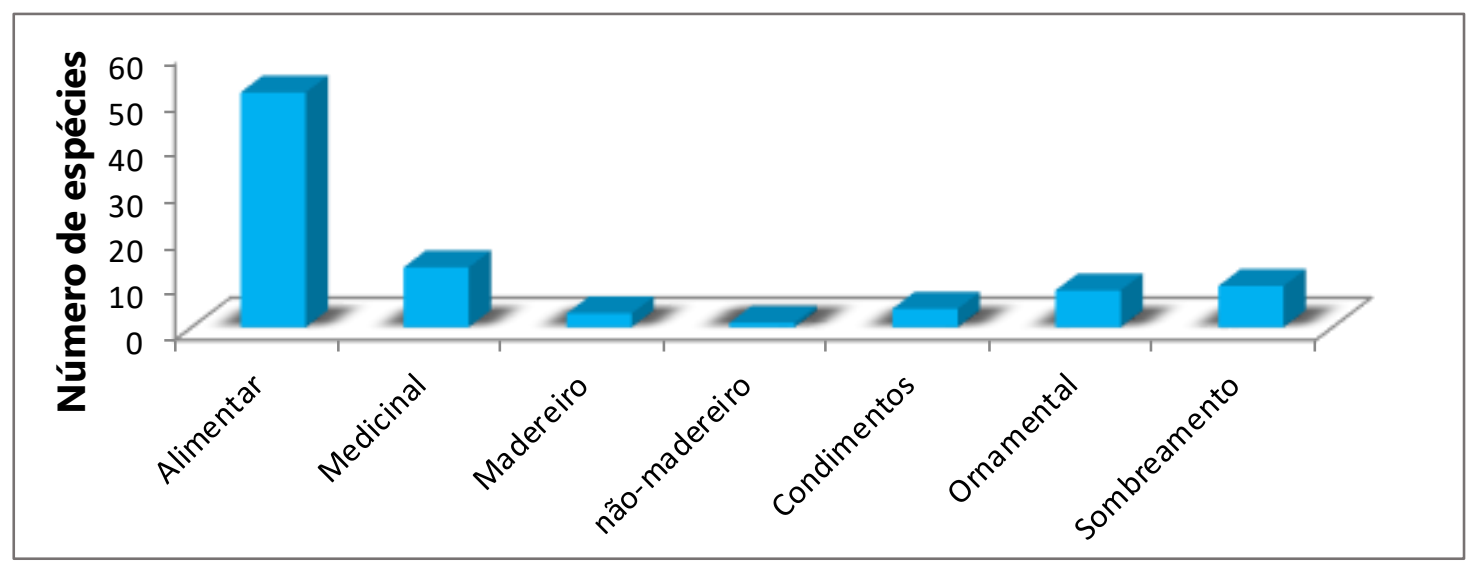

Usos

Fonte: Autor próprio.

Uma maior diversidade de espécies proporciona também uma melhor distribuiç̧ão no fornecimento de produtos ao longo do ano, minimizando os riscos de perda da produção e aumentando a segurança alimentar das famílias. De acordo com Trindade et al. (2010) no contexto da realidade amazônica, os quintais agroflorestais permitem que as populações locais obtenham fontes importantes de nutrientes para sua segurança alimentar, principalmente a partir dos alimentos ricos em proteínas, vitaminas e sais minerais.
Carneiro et al. (2013) em estudo sobre a importância dos quintais para a segurança alimentar dos agricultores no Ceará, verificou que a produção destes sistemas possibilita uma alimentação mais sadia às famílias, refletindo na melhoria da qualidade de vida.

Verificou-se que as famílias botânicas com maior abundância identificadas foram a Malvaceae com 142 indivíduos, a Musaceae e a Lamiaceae ambas com 80, Arecaceae com 77, a Euphorbiaceae 70, Poaceae 54 e Anacardiaceae com 53 (Figura 2). 
Figura 2. Distribuição das famílias mais abundantes no levantamento de diagnóstico dos quintais agroflorestais rurais no município de Parauapebas, PA, 2017.

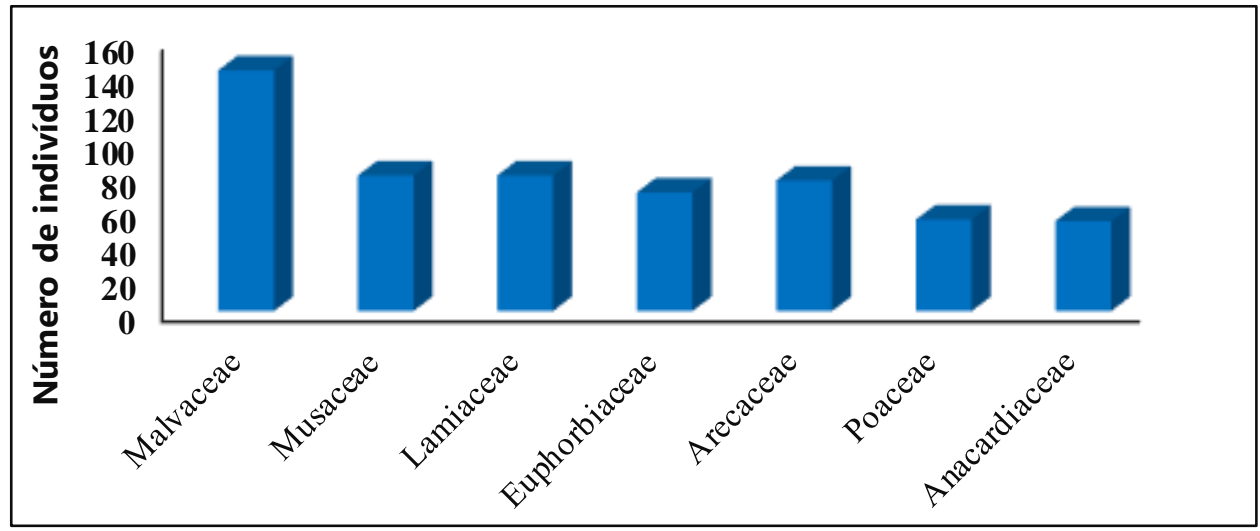

Famílias botânicas

Fonte: Dados da pesquisa

Em relação à abundância específica das espécies destacam-se: a Hibiscus sabdafira (100), Musa spp. (80), Manihot esculenta (68), Plectranthus barbatus (50), Saccharum spp. 48, Mangifera indica (35), Cocos nucifera (31), Allium fistulosum (30), Brassica oleracea (30), e Mentha villosa (30).

As espécies vinagreira (Hisbiscus sabdafira), a bananeira (Musa spp.) e a mandioca (Manihot esculenta), foram as espécies com maiores frequências, observadas em aproximadamente em $70 \%$ dos quintais estudados.

Segundo Vizotto e Pereira (2010) na região Nordeste do Brasil, as folhas de vinagreira são usadas no preparo de diversos pratos típicos da culinária, especialmente o "cuxá", além disso, de acordo com os autores esta espécie possui ações emolientes, diuréticas e sedativas. De acordo com Sousa et al. (2014) a vinagreira é bastante apreciada pelos nordestinos sendo que o "Arroz de cuxá", é um prato típico da culinária maranhense.

A bananeira (Musa spp.) é considerada importante pelos proprietários dos quintais, pois caracteriza-se por ser uma espécie que gera renda extra, contribuindo também para a subsistência da família. 
Já a mandioca conforme Pedro et al. (2013) é cultivada em todas as regiões do Brasil, assumindo importância na alimentação humana e animal, além de ser utilizada como matéria-prima em inúmeros produtos industriais.

$$
\text { O índice Shannon-Wiener }
$$
encontrado para os quintais agroflorestais estudados variou de 2,21 a 3,45 (Tabela 2).

Tabela 2. Índice de diversidade de espécies de Shannon-Wiener nos quintais agroflorestais, Parauapebas, PA, 2017.

\begin{tabular}{cc}
\hline Quintal & Índice de diversidade \\
\hline Quintal 1 & 2,21 \\
Quintal 2 & 3,15 \\
Quintal 3 & 2,48 \\
Quintal 4 & 2,98 \\
Quintal 5 & 3,45 \\
Quintal 6 & 3,39 \\
\hline
\end{tabular}

Fonte: Dados da pesquisa.

Figueiredo Júnior et al. (2013) realizando uma pesquisa em quintais agroflorestais em Anapú no Pará encontrou intervalo para o índice de Shannon-Wiener de 3,03. Já Silva et al. (2014) realizando levantamento em quintal agroflorestal em assentamento no município de Marabá obteve um índice médio de diversidade de aproximadamente 2,95. De acordo com Gliessman (2001) ecossistemas naturais relativamente diversificados apresentam índice de diversidade de Shannon entre 3 e 4 . Conforme os índices de diversidade verificados para os quintais agroflorestais visitados neste estudo, constata-se que houve uma diversidade considerável de espécies vegetais encontradas, Lima et al., (2000) afirmaram que índices de Shannon-Wiener elevados em geral relacionam áreas relativamente bem conservadas associadas a populações com significativo conhecimento etnobotânico.

Constatou-se que os quintais agroflorestais estudados do município de Parauapebas apresentam considerável diversidade de espécies, constituindo uma potencial fonte de alimentos para as famílias, este fato promove a segurança alimentar com qualidade do valor nutricional pela variedade de alimentos 
que enriquecem a dieta dos proprietários dos quintais.

As espécies de uso alimentar representaram a maioria da população de espécies vegetais total amostrada. No qual, as espécies vinagreira (Hisbiscus sabdafira), a bananeira (Musa spp.) e a mandioca (Manihot esculenta), foram as espécies com maiores frequências, observadas nos quintais estudados. Outro aspecto importante desses quintais agroflorestais é a conservação da biodiversidade local, o manejo e uso das plantas que possuem influência cultural e conhecimento empírico, tornando-se um bem social, principalmente sobre uso medicinal das plantas.

\section{REFERÊNCIAS}

ALBUQUERQUE, U. P.; LUCENA, R. F. P. Métodos e técnicas para coleta de dados. In: ALBUQUERQUE, U. P. (org.) Métodos e técnicas na pesquisa etnobotânica. Recife, Editora NUPEEA. p. 37-55. 2004.

AMARAL, C.N.; GUARIM NETO, G. Os quintais como espaços de conservação e cultivo de alimentos: um estudo na cidade de Rosário Oeste. Boletim do Museu Paraense Emílio Goeldi, Série Ciências Humanas, Belém, v. 3. N. 3 p. 329-341. 2008.
CARNEIRO, M. G. R.; CAMURÇA, A. M.; ESMERALDO, G.G.S.L.; SOUSA, N.R. Quintais Produtivos: contribuição à segurança alimentar e ao desenvolvimento sustentável local na perspectiva da agricultura familiar ( $\mathrm{O}$ caso do Assentamento Alegre, município de Quixeramobim/CE). Revista Brasileira de Agroecologia, v. 8, n. 2, p. 135-147, 2013.

CASTRO, A.P.; FRAXE, T. J. P.; SANTIAGO, J. L.; MATOS, R. B.; PINTO, I. C. OS sistemas agroflorestais como alternativa de sustentabilidade em ecossistemas de várzea no Amazonas. Acta Amazônica, v.39, n.2, p. 279-288, 2009.

FIGUEIREDO JÚNIOR, O.; HAMADA, M.O.S.; SOUZA, S.P.O.; CORREA, R.F. Levantamento florístico dos quintais agroflorestais do PDS Virola jatobá em Anapú, Pará. Enciclopédia biosfera, Centro Científico Conhecer, Goiânia, v.9, n.17; p. 1793, 2013.

GARCIA, R.; VIEIRA, T. A.; OLIVEIRA, F. A. de. Quintais agroflorestais e segurança alimentar em uma comunidade rural na Amazônia Oriental. Rev. Fac. Agron. V. 114, n. 1, p. 67-73 2015.

GAZEL FILHO, A. B. Composição, estrutura e função de quintais agroflorestais no Município de Mazagão, Belém, 2008. 104 f. Tese (Doutorado em Ciências Agrárias). Universidade Federal Rural da Amazônia, Embrapa Amazônia Oriental, Belém, 2008.

GLIESSMAN, S. R. Diversidade e estabilidade do agroecossistema. In:

Agroecologia: processos ecológicos em agricultura sustentável. Porto Alegre, Editora Universidade, 2001. p. 437-474. 
GOMES, G. S. Quintais agroflorestais no município de Irati-paraná, Brasil: agro biodiversidade e sustentabilidade socioeconômica e ambiental, 2010. $161 \mathrm{f}$. Tese (Doutorado em Ciências Florestais) Universidade Federal do Paraná. Setor de Ciências Agrárias. Paraná, 2008.

LIMA, R. X.; SILVA, S. M. KUNIYOSHI, Y. S.; SILVA, L. B. Etnobiologia de comunidades continentais da Área de Proteção Ambiental de Guaraqueçaba - Paraná, Brasil. Etnoecológica, v. 4, n. 6, p. 33-55, 2000.

PEDRO, L. P. M.; CUNHA, A. P. CARDOSO, E. L. M. Cultivo da Mandioca para o Estado do Pará. Disponível em: < http://sistemasdeproducao.cnptia.embra pa.br/FontesHTML/Mandioca/mandioca_ para/> . Acesso em 15 de jul. 2017.

PEREIRA, P. V. M.; FIGUEIREDO NETO, L. F. Conservação de espécies florestais: um estudo em quintais agroflorestais no município de Cáceres - MT. Revista Eletrônica em Gestão, Educação e Tecnologia Ambiental, v. 19, p. 783-793, 2015.

REZENDE, A. V. Diversidade, estrutura, dinâmica e prognose do crescimento de um cerrado senso stricto submetido a diferentes estudos por desmatamento, 2002. 243 f. Tese (Doutorado em engenharia florestal). Universidade Federal do Paraná, Curitiba, 2002.

RICKLEFS, R.E. A Economia da Natureza. $6^{a}$ ed. Editora Guanabara Koogan, Rio de Janeiro.2010.

SAVIERO, A.; DELUNARDO, T. A.; HAVERROTH, M.; OLIVEIRA, L. C.; MENDONÇA, A. M. S. Cultivo de Espécies
Alimentares em Quintais Urbanos de Rio Branco, Acre, Brasil. Revista Acta Botânica Brasílica, v. 25, n. 3, p. 549-556, 2011.

SILVA, T. P. da. Levantamento de espécies vegetais e utilização em quintal agroflorestal de estabelecimento agrícola no assentamento alegria - Marabá, Pará. Agroecossistemas, v. 6, n. 1, p. 103-109, 2014.

SIQUEIRA, G. W.; APRILE, F.; MIGUÉIS, A. M. Diagnóstico da qualidade da água do rio Parauapebas (Pará - Brasil). Acta Amazônica. vol. 42, n. 3, p. 413 - 422, 2012.

SOUSA, D. A.; OlIVEIRA, A. A. de. GONÇALO, M. C. da. Agrobiodiversidade em quintais familiares no município de Caxias no Maranhão. ENCICLOPÉDIA BIOSFERA, Centro Científico Conhecer, v.10, n.18, p. 3129, 2014.

TRINDADE, E. F. S.; REBELLO, F. K.; KATO, O. R. Agroecossistemas como alternativa de desenvolvimento na Amazônia: experiências em comunidades rurais no nordeste paraense (PA). In: VIII Congresso Latino-Americano de Sociologia Rural, Porto de Galinhas, 2010. Anais eletrônicos... Porto de Galinhas: CLASR, 2010. Disponível em: <http://www.alasru.org/wpcontent/uploads/2011/07/GT2-

ElineuzaTRINDADE REBELLOKATO_28maio2010.pdf> Acesso em: 15 jul. 2017.

VIZZOTO, M.; PEREIRA, M.C. Hibisco: do uso ornamental ao medicinal. 2008. Disponível em: <http://www.infobibos.com/Artigos/2008 _4/hibisco/index.htm>. Acesso em: 15 jul. 2017. 